\title{
Variations
}

Variations

Revue internationale de théorie critique

$17 \mid 2012$

Critique du travail

\section{Repenser Le Capital à la lumière des Grundrisse}

\section{Moishe Postone}

Traducteur : Julien Bordier

\section{OpenEdition}

\section{Journals}

Édition électronique

URL : http://journals.openedition.org/variations/382

DOI : 10.4000/variations.382

ISSN : 1968-3960

Éditeur

Les amis de Variations

Référence électronique

Moishe Postone, "Repenser Le Capital à la lumière des Grundrisse », Variations [En ligne], 17 | 2012, mis en ligne le 15 octobre 2012, consulté le 19 avril 2019. URL : http://journals.openedition.org/

variations/382 ; DOI : 10.4000/variations.382

Ce document a été généré automatiquement le 19 avril 2019.

Les ami•e•s de Variations 


\title{
Repenser Le Capital à la lumière des Grundrisse
}

\author{
Moishe Postone
}

Traduction : Julien Bordier

\section{NOTE DE L'ÉDITEUR}

Pour rester proche de l'argumentation de l'auteur, les extraits cités ici sont re-traduits à partir du texte en anglais. L'ensemble du jeu de référence est laissé dans sa version originale, les références bibliographiques renvoyant aux versions citées par l'auteur. Pour une version française des Grundrisse voir la réédition récente : Karl Marx, Manuscrits de 1857-1858 dits Grundrisse, 2011, Paris, La Dispute/Editions Sociales, 929 p. Pour une version française de l'ouvrage majeur de l'auteur voir : Moishe Postone, Temps, travail et domination sociale, 2009, Paris, Mille et Unes Nuits, 587 p.

Variations remercie les éditions Routledge et Marcello Musto de nous permettre de publier cette traduction inédite en français. Ce texte est extrait de l'ouvrage collectif Karl Marx's Grundrisse - Foundations of the critique of political economy 150 years later, dirigé par Marcello Musto (2020, London, Routledge, 292 p.), http://www.routledge.com/books/ details/9780415588713.

\section{NOTE DE L'AUTEUR}

Remerciements à Robin Bates et Jake Smith pour leurs retours critiques.

\section{Théorie critique de la société et monde contemporain}

1 La théorie critique de la société semble avoir été dépassée par les transformations globales des trois dernières décennies. L'intense et fructueux renouveau de la pensée et des enseignements marxiens dans les années 60 et 70 fut suivi d'un profond désintérêt de 
la part de nombreux théoriciens. L'espace intellectuel devint dominé par les approches postmodernes et poststructuralistes, qui apparaissaient pour certains comme des critiques viables du marxisme. Pourtant, il semble de plus en plus évident que de telles approches ne saisissent pas de manière adéquate l'époque actuelle. Elles échouent à élucider les changements historiques de base qui ont reconfiguré le monde durant ces dernières décennies. Même des penseurs majeurs tels que Habermas, Foucault et Derrida apparaissaient désormais comme les théoriciens d'une configuration historique finissante - le fordisme déclinant. Leurs approches critiques éclairent de moins en moins l'univers social contemporain.

2 Une des faiblesses évidentes de ces discours post-marxistes a été l'absence d'une prise en compte sérieuse de l'économie politique, une absence devenue flagrante face à la globalisation. Dans le même temps, si importante que soit l'intégration des problématiques de l'économie politique dans les théories critiques du monde contemporain, il est clair qu'un retour au marxisme traditionnel n'est pas pertinent. Cette grille de lecture critique traditionnelle a échoué à fournir les bases d'une analyse historique des régimes communistes d'accumulation. Ses présupposés d'économie politique ont été défiés sur la base de l'importance croissante du savoir scientifique et des technologies de pointe dans le processus de production. Enfin, ses idéaux d'émancipations se sont progressivement éloignés des thématiques des mécontentements sociaux et culturels les plus courants.

3 Toutefois, les tendances historiques les plus récentes suggèrent l'importance d'une théorie critique du capitalisme adéquate. Bien que ces tendances incluent une réflexion sur le caractère anachronique du marxisme traditionnel - par exemple l'émergence des nouveaux mouvements sociaux tels que les mouvements écologistes de masse, les mouvements de femmes, les mouvements gay, les mouvements pour l'émancipation des minorités, ainsi que les mécontentements exprimés par de nombreux mouvements «fondamentalistes »- les dernières décennies se sont aussi caractérisées par la réémergence d'un éclatement économique mondial et l'intensification des rivalités intercapitalistes à une échelle globale. Ces évolutions suggèrent qu'une analyse critique adéquate au monde contemporain doit être en mesure de saisir à la fois de nouvelles dimensions significatives et la continuité sous-jacente propre au capitalisme.

Les Grundrisse der Kritik der politischen Ökonomie de Marx pourraient fournir le point de départ d'une analyse critique stimulante basée sur une nouvelle réflexion fondamentale sur la nature même du capitalisme (Marx 1973). Ecrits en 1857 et 1858, ces manuscrits furent publiés pour la première fois en 1939 et ne connurent pas de renommée plus large avant la fin des années 60 et le début des années 70. Bien que Marx n'élabora pas, dans les Grundrisse, l'ensemble des aspects ultérieurs de sa critique, le fond général de sa critique de la modernité capitaliste, ainsi que la nature et la signification des catégories fondamentales de sa critique y apparaissent très clairement. Le Capital est plus difficile à déchiffrer et sa lecture peut mener à des incompréhensions tant il est structuré comme une critique immanente - une critique amorcée à partir d'un point de vue immanent à son objet d'investigation. Pour cette raison, ses catégories peuvent être incomprises et perçues comme positives plutôt que critique. Ainsi, bien trop souvent, l'objet de la critique de Marx est interprété comme étant son point de vue - ce sur quoi nous reviendrons. Ce problème se pose de manière moins pressante à la lecture des Grundrisse qui ne sont pas structurés de manière rigoureuses. Parce que Marx était encore en train d'y former son analyse catégorielle ${ }^{1}$. Son intention stratégique y est plus accessible que dans Le Capital. 
Ainsi, les Grundrisse peuvent éclairer la nature et le fond de la critique de l'économie politique des écrits ultérieurs de Marx. Quand elle est lue à travers le prisme des manuscrits de 1857-1858, cette critique peut fournir la base d'une théorie critique du monde contemporain plus adéquate que celle élaborée à travers le cadre du marxisme traditionnel $^{2}$.

\section{Le marxisme traditionnel}

5 Avant de développer cette idée en s'appuyant sur des sections essentielles des Grundrisse, je vais d'abord décrire brièvement ce que j'entends par « marxisme traditionnel. » Cela ne fait pas référence à une tendance historique spécifique du marxisme, mais de manière plus générale à toute analyse du capitalisme faite essentiellement en termes de relations de classe, enracinées dans la propriété privée et médiatisées par le marché. Les relations de dominations y sont comprises essentiellement en termes de domination de classe et d'exploitation. Dans cette approche interprétative générale, le capitalisme est caractérisé par la contradiction structurelle grandissante entre les relations sociales de base régissant la société (interprétées comme étant la propriété privée et le marché) et les forces de production (interprétées comme étant le mode de production industriel). Le socialisme, quant à lui, est compris en termes de propriété collective des moyens de production et de planification centralisée, dans un contexte industrialisé. Il est donc conceptualisé comme un mode de distribution régulé, conscient et juste, qui conviendrait au mode de production industriel (qui est lui-même compris comme un processus technique intrinsèquement indépendant du capitalisme).

6 Cet état de compréhension général est lié à une compréhension particulière des catégories de base de la critique de l'économie politique élaborée par Marx. Sa catégorie de valeur, par exemple, a été généralement interprétée comme une manière de montrer que le travail humain direct crée de la richesse sociale partout et toujours, une richesse qui se retrouve médiatisée par le marché dans le capitalisme. Sa théorie de la plus-value [surplus value], dans une telle approche, démontre l'existence de l'exploitation dans le capitalisme en montrant que le travail seul crée le surplus de production, qui est ensuite approprié par la classe capitaliste ${ }^{3}$.

7 Cette interprétation est basée sur une compréhension transhistorique du travail comme étant une activité de médiation entre la nature et les hommes, transformant la matière d'une manière motivée par un but, et qui est une condition de la vie sociale. Le « travail », ainsi interprété, est perçu comme la source de richesse dans toutes les sociétés et comme étant ce qui constitue ce qui est universellement et authentiquement social. Mais, dans le capitalisme, la pleine réalisation du «travail» est entravée par des relations de fragmentation et particularisées. À partir de là, l'émancipation se réaliserait dans une forme sociale où le «travail » transhistorique, libéré des déformations imposées par le marché et la propriété privée, s'affirmerait comme étant le principe de régulation de la société. (Cette idée, bien évidemment, est soumise au fait que la révolution socialiste soit l'« auto-réalisation » du prolétariat.) Ici, le «travail », fourni le point de départ de la critique du capitalisme.

8 À partir du cadre de base du "marxisme traditionnel ", ainsi conceptualisé, toute une variété d'approches théoriques, méthodologiques et politiques ont été construites ${ }^{4}$. Pourtant, bien que des analyses économiques, politiques, sociales, historiques et culturelles puissantes aient été élaborées à partir de cette grille de lecture, ses limites 
sont depuis longtemps manifestes au regard de l'évolution historique au XXe siècle. Dépasser l'inévitable centralité du capitalisme dans le monde d'aujourd'hui, nécessite alors une reconceptualisation du capital qui rompe avec le cadre du marxisme traditionnel.

Rétrospectivement, il est devenu évident que la configuration sociale/politique/ économique/culturelle de l'hégémonie du capital a historiquement évolué - du mercantilisme au capitalisme libéral du XIXe siècle, du capitalisme fordiste centré sur l'Etat du XXe au capitalisme global néo-libéral contemporain. Cela implique que le capitalisme ne peut être entièrement identifié à partir d'une de ses configurations historiques et cela pose la question de la nature du noyau fondamental du capitalisme en tant que forme de vie sociale, c'est-à-dire, de la nature même du capital.

\section{Les Grundrisse : le noyau du capitalisme}

10 Les Grundrisse aident à clarifier la conception ultérieure de Marx quant au noyau du capitalisme, ainsi que la nature de son succès historique, de manière beaucoup plus approfondie que l'interprétation marxiste traditionnelle. Dans une section essentielle du manuscrit «Contradiction entre la fondation de la production bourgeoise (la valeur comme mesure) et son développement», Marx indique explicitement ce qu'il perçoit comme étant le noyau du capitalisme et la contradiction fondamentale qui implique la possibilité historique d'une forme de vie sociale postcapitaliste. Il commence ce chapitre en écrivant que « l'échange de travail vivant contre du travail objectivé - c'est-à-dire la place du travail social en fonction de la forme de la contradiction du capital et du travail salarié - est le développement ultime de la relation de valeur et de la production reposant sur la valeur. » (Marx 1973 : 704). Le titre et la phrase d'ouverture de ce chapitre des Grundrisse indique que, pour Marx, la catégorie de valeur explique la base des relations de production dans le capitalisme - ces relations sociales qui caractérisent profondément le capitalisme en tant que forme de vie sociale. Dans le même temps, la catégorie de valeur exprime une forme de richesse déterminée. Il s'ensuit qu'une analyse de la valeur doit élucider ces deux aspects. En tant que forme de richesse, la valeur a généralement été comprise comme une catégorie de médiation, par le marché, de la richesse créée par le travail. Ainsi, quand Marx évoque dans le passage cité "l'échange " en jeu dans la « relation de valeur ", l'échange ne se réfère pas à la distribution mais à la production « l'échange de travail vivant contre du travail objectivé. » Chez Marx, la caractérisation de la valeur en tant que «fondation de la production bourgeoise » indique qu'elle ne doit pas être comprise simplement comme une catégorie du mode de distribution des marchandises, ou une critique du marché soit-disant auto-régulé. Elle doit plutôt être comprise avant tout comme une catégorie de la production capitaliste elle-même.

Dans les Grundrisse donc, l'analyse de la contradiction entre les "relations de production » et « les forces de production » dans le capitalisme diffère de celle proposée par les théories marxistes traditionnelles. Ces dernières se concentrent sur une critique du mode de distribution (le marché, la propriété privée) et perçoivent cette contradiction comme l'antagonisme entre les sphères de distribution et de production. Marx critique explicitement les approches théoriques qui conceptualisent le changement historique en termes de mode de distribution, sans considérer la transformation potentielle du mode de production. C'est pourquoi il cite, pour les illustrer, John Stuart Mill qui affirmait : « les lois et conditions de la production de richesse ont des caractéristiques de vérités 
physiques (...). Il n'en va pas de même avec la distribution de la richesse. Celle-ci n'est qu'une question d'institutions humaines..$^{5} »$ Cette séparation est, selon Marx, illégitime : "Les "lois et conditions" de la production de richesse et les lois de "la distribution de richesse" sont les mêmes lois sous des formes différentes, elles évoluent ensemble, suivant le même processus historique » (Marx 1973 : 832).

Si le processus de production et les relations sociales fondamentales dans le capitalisme interagissent, le premier ne peut pas être confondu avec les forces de productions, qui peuvent potentiellement entrer en contradiction avec les relations de production capitalistes. Mais il convient par contre de considérer le processus de production luimême comme étant intrinsèquement lié au capitalisme. En d'autres termes, ce que Marx entend comme la contradiction fondamentale du capitalisme n'est pas celle entre la production industrielle d'un côté, et le marché et la propriété privée de l'autre. Cela mérite d'être approfondi.

Quand il présente la production reposant sur la valeur, il la décrit comme un mode de production dont « la présupposition est - et reste - la masse de temps de travail direct, la quantité de travail employée, comme le facteur déterminant dans la production de richesse » (Marx 1973 : 704 ; je souligne). Ce qui caractérise la valeur en tant que forme de richesse, selon Marx, est ce qui est constitué par la dépense du travail humain direct dans le processus de production, mesurée temporellement. La valeur est une forme sociale qui exprime, et qui est basée sur, l'extension du temps de travail direct. Cette forme, toujours pour Marx, constitue précisément le cœur du capital. En tant que catégorie des relations sociales fondamentales qui constituent le capitalisme, la valeur exprime ce qui est, et reste, la fondation de base de la production capitaliste. Alors, la production basée sur la valeur génère une dynamique qui accentue la tension croissante entre cette fondation $\mathrm{du}$ mode de production capitaliste et les conséquences de sa propre évolution historique :

Mais dans la mesure où l'industrie se développe, la création de richesse réelle en vient à moins dépendre du temps de travail et de la quantité de travail employé, que sur le pouvoir des agents en mouvements pendant le temps de travail, dont la " puissante efficacité » est elle-même (...) non proportionnelle au temps de travail direct dépensé pour leur production, mais dépend plutôt de l'état général de la science et du progrès technique. (...) La richesse réelle se manifeste plutôt (...) dans la disproportion monstrueuse entre le temps de travail dépensé et son produit, autant que dans le déséquilibre qualitatif entre le travail, réduit à une pure abstraction, et le pouvoir du processus de production qu'il supervise. (Marx 1973, 704-5)

Le contraste entre valeur et « richesse réelle » se manifeste entre une forme de richesse basée sur le «temps de travail et la quantité de travail employé » et une autre qui ne dépend pas directement du temps de travail. Ce contraste est essentiel pour comprendre la théorie de la valeur de Marx et sa notion de contradiction de base de la société capitaliste. Cela va encore plus loin : la valeur n'est pas qu'une catégorie du marché, qui décrirait un mode de distribution sociale de la richesse historiquement spécifique. Une telle interprétation centrée sur la marché - qui est liée à la position de Mill sur la possibilité de transformation historique du mode de distribution mais pas de celui de production - implique l'existence d'une forme transhistorique de richesse distribuée différemment dans des sociétés différentes. Pourtant, selon Marx, la valeur est une forme spécifique de richesse sociale, intrinsèquement liée à un mode de production historiquement spécifique. Cela suggère que des formes de sociétés différentes impliquent des formes de richesse différentes. (L'argumentation de Marx à ce sujet 
suggère que la forme de richesse, la forme du travail et la base des relations sociales diffèrent dans de nombreuses sociétés.)

De nombreuses discussions sur l'analyse par Marx de la place centrale du travail comme source de valeur - qu'elles y adhèrent ou qu'elles la critiquent - ignorent la distinction faite entre la « richesse réelle » (ou « richesse matérielle ») et la valeur. Les Grundrisse, au contraire, indiquent que la «théorie de la valeur par rapport au travail » n'est pas une théorie des propriétés propres au travail en général. Elle est une analyse de la spécificité historique de la valeur comme forme de richesse et donc, implicitement, une analyse du travail qui la constitue. Par conséquent, il est inutile de débattre pour ou contre la théorie de la valeur de Marx comme si elle était une théorie de la richesse (transhistorique) par rapport au travail - c'est-à-dire comme si Marx avait écrit un traité d'économie politique plutôt qu'une critique de l'économie politique ${ }^{6}$. Cela ne signifie pas, bien-sûr, que l'interprétation de la catégorie de valeur de Marx en tant que catégorie historiquement spécifique valide toute son analyse de la société moderne. Mais cela pousse à considérer l'analyse de Marx dans ses propres termes historiques, et non pas comme si elle était une théorie transhistorique d'économie politique, telle que celle qu'il a fortement critiqué.

Ces réflexions suggèrent que la valeur, saisie dans la lecture de Marx, est une catégorie critique qui révèle la spécificité historique des formes de richesse et de production qui caractérisent le capitalisme. Le passage cité plus haut montre que, selon Marx, la forme de production basée sur la valeur se développe d'une manière qui tend à la possibilité de la négation historique de la valeur elle-même. Dans une analyse qui semble bien en phase avec la situation contemporaine, Marx argumente qu'à mesure que la production industrielle capitaliste se développe, la valeur devient de moins en moins adéquate pour mesurer la richesse sociale. Il pointe alors le contraste entre la valeur, qui est une forme de richesse soumise à l'utilisation du temps de travail humain, et le gigantesque potentiel de production de richesse de la science et de la technologie modernes. La valeur devient anachronique par rapport au potentiel du système de production qu'elle sous-tend. La réalisation de ce potentiel entraînerait l'abolition de la valeur.

Cette possibilité historique ne signifie pas pour autant que la toujours plus grande masse de biens produits pourrait être fabriquée sur la base du mode de production industriel existant, et distribué de manière plus équitable. La logique de la contradiction entre la «richesse réelle» et la valeur, qui tend vers la possibilité que le premier dépasse le second comme forme déterminante de la richesse sociale, implique également la possibilité d'un mode de production différent. Celui-ci serait alors basé sur une structure renouvelée et davantage émancipatrice du travail social :

Le travail ne semble plus vraiment faire partie du processus de production; au contraire, l'être humain devient davantage un surveillant et un régulateur de ce processus. (...) Il se retire du processus de production au lieu d'en être l'acteur principal. Dans cette transformation, ce n'est ni le travail humain direct qu'il réalise lui-même, ni le temps pendant lequel il travaille, mais plutôt l'appropriation de son propre pouvoir productif en général, sa compréhension de la nature et de sa maîtrise par lui-même comme un ensemble social - c'est, en un mot, le développement de l'individu social qui apparaît alors comme la pierre angulaire de la production et de la richesse. Le vol du temps de travail de l'autre, sur quoi la richesse actuelle est basée, apparaît comme une base misérable comparée à cette nouvelle fondation, crée par la grande industrie elle-même. (Marx 1973 : 705 ; je souligne le deuxième passage) 

l'abolition de la valeur comme forme sociale de richesse, ce qui implique à son tour le dépassement du mode de production déterminé développé par le capitalisme. Le temps de travail ne servirait plus à mesurer la richesse, et la production de richesse ne serait plus créée en premier lieu par le travail humain direct dans le processus de production : «Dès que le travail dans sa forme directe a cessé d'être le grand créateur de richesse, le travail humain cesse et doit cesser d'être sa mesure, et donc, la valeur d'échange d'être la mesure de la valeur d'usage. » (Marx $1973:$ 705) richesse, et sa forme matérielle de production, comme étant interconnectées: la production reposant sur la valeur, le mode de production construit sur le travail salarié et la production industrielle basée sur le travail prolétarien sont intrinsèquement reliés, dans cette analyse. Ainsi, le caractère toujours plus anachronique de la valeur signifie également le caractère toujours plus anachronique du processus industriel de production développé dans le capitalisme. Le capitalisme avancé, selon Marx, mène à une transformation fondamentale de la forme matérielle de la production, de la manière dont les gens travaillent.

Malgré cela, la société socialiste, toujours pour Marx, n'émerge pas automatiquement telle la conséquence d'un développement historique linéaire et évolutionniste. La transformation radicale du processus de production mentionnée plus haut n'est pas le résultat quasi-automatique du développement rapide des savoirs techniques et scientifique et de leurs applications. C'est plutôt une possibilité qui naît d'une contradiction sociale intrinsèque croissante. Bien que la course du développement capitaliste génère la possibilité d'une structure nouvelle et émancipatrice du travail social, sa réalisation générale est impossible dans le capitalisme.

Le capital lui-même est la contradiction en mouvement, en cela il pousse à réduire le temps de travail au minimum, tandis qu'il place le temps de travail, d'un autre côté, comme l'unique source et mesure de richesse. Ainsi il diminue le temps de travail dans sa forme nécessaire pour l'augmenter dans sa forme superflue, et le superflu se présente de plus en plus comme une condition - une question de vie ou de mort - pour le nécessaire. (Marx 1973 : 706)

La question du temps de travail " nécessaire » et "superflu » ne peut être entièrement développée ici. Mais il est important de noter que, selon Marx, bien que le capitalisme tend à développer de puissantes forces de production dont le potentiel rend toujours plus obsolète une organisation de la production basée sur la dépense de temps de travail direct, sa structure est telle qu'elle ne peut permettre la pleine réalisation de ce potentiel. La seule forme de richesse qui constitue le capital est celle basée sur la dépense de temps de travail direct. Ainsi, malgré la différence croissante entre la valeur comme mesure et la richesse matérielle, la valeur n'est pas simplement remplacée par une nouvelle forme de richesse ${ }^{7}$. Au contraire, elle reste pour Marx la précondition structurelle nécessaire de la société capitaliste (bien qu'il argumente dans Le Capital que ce n'est pas ouvertement le cas).

22 Sur la base de ses catégories de valeur, marchandise et capital, Marx montre que le capitalisme se caractérise par une dynamique de développement intrinsèque. Mais cette dynamique reste soumise au capitalisme, elle ne se réalise pas d'elle-même. Les catégories éclairent autant cette dynamique que ses limites: ce qui devient "superflu» dans la production de richesse matérielle reste structurellement « nécessaire » pour le capital. Le 
capitalisme porte véritablement la possibilité de sa propre négation, mais il n'évolue absolument pas automatiquement en quelque chose d'autre. Que la dépense de temps de travail humain direct reste centrale et indispensable pour le capital, alors qu'elle est rendue anachronique à cause des développements générés par le capital, fait surgir une tension interne. Comme je l'ai montré dans Temps, travail et domination sociale, c'est à partir de cette tension que Marx analyse la nature de la production industrielle et sa trajectoire de développement.

Ces extraits des Grundrisse indiquent que la notion de Marx d'une contradiction structurelle dans le capitalisme ne devrait pas être immédiatement ramenée à un antagonisme social tel que le conflit de classes. Ils révèlent également que la compréhension de la contradiction capitaliste par Marx ne se réfère pas de manière fondamentale à une opposition entre l'appropriation privée et la production socialisée ${ }^{8}$. En cela, ses analyses sont fondamentalement différentes de celles du marxisme traditionnel. Marx n'analyse pas la contradiction du capitalisme, dans les Grundrisse, comme étant celle entre le processus de production et la valeur, c'est-à-dire, entre la production dans le capitalisme et les relations sociales capitalistes. Au contraire, il traite le premier comme étant moulé dans le second: la production dans le capitalisme est le « mode de production basé sur la valeur. » C'est en ce sens que, dans ses écrits ultérieurs, Marx évoque explicitement le mode de production industriel comme étant une «forme spécifiquement capitaliste de production (...) (et aussi technologiquement) » (Marx 1973 : 428). Ces passages des Grundrisse impliquent que la forme matérielle de la production doit être transformée dans le capitalisme avancé. Ils démentent également l'idée que la théorie critique de Marx serait une forme d'évolutionnisme technologique déterministe 9 $\mathrm{Au}$ contraire, il considère la technologie et le processus de production comme étant socialement constitués; tous deux sont formés par la valeur. Ils ne devraient donc pas être simplement identifiés aux «forces de productions » qui entrent en contradiction avec les relations sociales capitalistes. Pourtant, bien que la technologie et le processus de production soient moulés par les relations capitalistes, ils donnent corps à une contradiction. L'analyse de Marx distingue la réalité de la forme de production constituée par la valeur et son potentiel - un potentiel qui appelle la possibilité d'une autre forme de production. Cette distinction est enracinée, au final, dans la nature contradictoire des relations capitalistes, que Marx, dans Le Capital, situe dans le double caractère des catégories de la vie sociale moderne et capitaliste.

Il est évident à partir des passages cités que quand, dans les Grundrisse, Marx décrit le dépassement de la contradiction du capitalisme et écrit que «la masse des travailleurs doit elle-même s'approprier le surplus de son propre travail» (Marx 1973 : 708), il ne se réfère pas seulement à l'expropriation de la propriété privé et à l'utilisation du surplus produit dans d'une manière plus rationnelle, juste et efficace. L'appropriation qu'il évoque implique aussi l'application réflexive du potentiel propre à la production capitaliste avancée dans le processus de production lui-même. Le système de production social par lequel la richesse est créée grâce à l'appropriation du temps de travail direct, et, à l'activité des travailleurs traités tels les engrenages d'un appareil de production, pourrait être aboli. Pour Marx, ces deux aspects du mode de production capitaliste sont liés. Ainsi, le capitalisme avancé, tel qu'il est présenté dans les Grundrisse, implique implicitement le dépassement des aspects matériels et formels du mode de production basé sur le travail salarié. Cela mène à l'abolition d'un système de distribution construit sur l'échange de force de travail, comme marchandise contre un salaire, avec lequel les 
biens de consommations sont acquis. Cela mène également à l'abolition d'un système de production basé sur le travail prolétarien, c'est-à-dire, sur le travail fragmenté et unilatéral, caractéristique de la production industrielle capitaliste. Par rapport à la structure du travail social, la contradiction marxienne doit donc être comprise comme une contradiction croissante entre la sorte de travail effectué par les gens dans le capitalisme, et la sorte de travail qu'ils pourraient effectuer si la valeur était abolie et si le potentiel productif développé par le capitalisme était réflexivement utilisé pour libérer les gens du contrôle des structures aliénées construites par leur propre travail. Loin de mener à la réalisation du prolétariat, le capitalisme avancé implique l'abolition matérielle du travail prolétarien. L'émancipation $d u$ travail requiert l'émancipation contre le travail (aliéné).

Cette interprétation, en fournissant la base pour une critique historique des formes concrètes de production dans le capitalisme (mais aussi, bien-sûr, de la médiation et de la domination abstraites exprimées par les catégories de valeur et de capital) met en lumière l'affirmation bien connue de Marx selon laquelle la formation sociale capitaliste marque la fin de la préhistoire de la société humaine (Marx 1987: 264). L'idée d'une généralisation du travail prolétarien implique que cette " préhistoire » doit être comprise comme une référence aux formations sociales dans lesquelles le surplus continu de production existe mais est basé en premier lieu sur le travail humain direct. Cette caractéristique se retrouve dans les sociétés dans lesquelles le surplus est créé par l'esclave, le serf, ou le travail salarié. Mais la formation basée sur le travail salarié, pour Marx, est la seule à être caractérisée par une dynamique qui offre la possibilité historique que le sur-produit basé sur le travail humain, comme élément interne au processus de production, puisse être dépassé. Une nouvelle formation sociale peut alors être créée, dans laquelle «le surplus de travail de la masse a cessé d'être la condition de développement de la richesse générale, tout comme le non-travail d'un petit nombre, au profit du développement des pouvoirs généraux de la tête humaine " (Marx 1973 : 705).

Pour Marx donc, la fin de la préhistoire signifie l'avènement de l'opposition entre les travaux manuel et intellectuel. Pourtant, cette opposition ne peut être dépassée en mélangeant simplement le travail manuel et le travail intellectuel. La vision de la production par Marx dans les Grundrisse implique non seulement que la séparation de ces deux modes de travail, mais aussi que les caractéristiques déterminantes de chacun, sont enracinées dans la forme de production existante. Leur séparation pourrait être dépassée en transformant les modes existants des travaux manuel et intellectuel, c'est-à-dire, par la construction d'une nouvelle structure et organisation du travail. Une telle structure renouvelée devient possible, selon l'analyse de Marx, quand la production du surplus n'est plus basée nécessairement et avant tout sur le travail humain direct.

La section des Grundrisse sur la contradiction fondamentale du capitalisme indique donc que la théorie critique de Marx doit être comprise essentiellement comme une critique $d u$ travail dans le capitalisme ; plutôt qu'une critique du capitalisme du point de vue du travail (comme le fait le marxisme traditionnel). Cela a d'importantes conséquences dans la compréhension du Capital et trace une distinction fondamentale entre la critique de l'économie politique de Marx et sa fréquente (in)compréhension comme une économie politique critique. Élaborer pleinement une telle lecture du Capital sur la base des Grundrisse n'est bien-sûr pas possible avec le cadre proposé par cette argumentation. Toutefois, pour pouvoir esquisser ses contours, il est important de considérer une autre 
section essentielle des Grundrisse intitulée «La méthode de l'économie politique » (Marx $1973: 100-8)$.

\section{Les Grundrisse : les catégories de Marx}

Dans cette section, Marx s'efforce de trouver un point de départ adéquat à son analyse critique. Il rend évident que les catégories de son analyse ne devraient pas être comprises dans des termes économiques étroits. Au contraire, elles « expriment les formes d'être [Daseinsformen], les déterminations de l'existence [Existenzbestimmungen] (...) de cette société spécifique» (Marx 1973 : 106). En tant que telles elles sont, dans un premier temps, autant des formes de la subjectivité et de l'objectivité ; elles expriment « ce qui est donné, aussi bien dans la tête que dans la réalité » (Marx 1973 : 106). Cela signifie que les catégories de Marx proposent de saisir comment les dimensions économiques, sociales, et culturelles de la vie moderne et capitaliste sont intrinsèquement inter-reliées, alors qu'elles sont généralement traitées comme liées les unes autres autres de manière extrinsèque et contingente. Cette approche des catégories contredit la compréhension des relations entre l'objectivité et la subjectivité sociales en termes de infra/ superstructure $^{10}$.

Plus encore, Marx insiste sur le fait que les catégories de sa critique sont historiquement spécifiques. Même les catégories qui semblent être transhistoriques et qui jouent bien un rôle dans des périodes historiques antérieures - telles que l'argent et le travail - sont pleinement développées et ne deviennent vraiment elles-mêmes que dans la société capitaliste (Marx 1973 : 103).

L'exemple du travail montre de manière frappante comment les catégories les plus abstraites, en dépit de leur validité (...) dans toutes les époques, sont toutefois, dans le caractère spécifique de cette abstraction, elles-mêmes tels le produit des relations historiques, et ne possèdent pleinement leur validité seulement dans et pour ces relations. (Marx 1973 : 105)

En d'autres termes, aussi simplement «modernes » que soient les catégories abstraites « autant sont les relations qui créent cette abstraction simple » (Marx $1973: 103)^{11}$.

Puisque les catégories abstraites, pleinement développées, sont historiquement spécifiques,

il serait (...) infaisable et faux de laisser les catégories économiques s'enchaîner les unes les autres dans la même suite que dans laquelle elles furent historiquement décisives. Leur enchaînement est en fait déterminé par leurs relations dans la société bourgeoise moderne, ce qui est précisément l'opposé de ce à quoi (...) correspond le développement historique. (Marx 1973 : 107)

Au contraire, l'analyse critique doit partir de ce qui est essentiel pour son objet. Dans la société bourgeoise, «le capital est le pouvoir économique tout puissant » et partant, il « doit être le point de départ tout comme l'objectif. » (Marx 1973 : 107)

L'insistance de Marx sur la spécificité historique de son objet d'investigation est intrinsèquement lié à la question du point de départ de son analyse critique. Comme plus tôt dans L'idéologie allemande, Marx insiste sur la construction sociale et historique des formes de conscience, ceci est affiné dans les Grundrisse avec la référence à la notion du caractère objectif/subjectif des catégories structurantes de la société capitaliste. Cela implique qu'aucune position, y compris celle de Marx, n'a de signification transhistorique et universelle. La relativisation historique de la pensée ne signifie pas pour autant qu'une 
théorie valide soit impossible. Une théorie historiquement spécifique peut être rigoureusement adéquate à son objet. Cela nécessite que la théorie soit auto-réflexive : elle doit être capable d'appliquer à ses propres conditions de possibilité au moyen des mêmes catégories qui lui permettent de saisir son objet, c'est-à-dire, son propre contexte. Plus encore, le caractère historiquement spécifique de la théorie n'est pas qu'une question de contenu, mais aussi une question de forme. Sa forme ne doit pas contredire le caractère historiquement spécifique de la théorie. Par exemple, elle ne peut se présenter comme étant une forme transhistorique, comme une « méthode » universellement valide qui pourrait s'appliquer à des objets différents, auxquels elle n'est liée que de manière contingente. Au contraire, la spécificité historique de la théorie demande à ce que le concept soit propre à son objet. (Ironie du sort, c'est quand la théorie est auto-consciente et réflexivement spécifique historiquement que la sentence hégélienne, apparemment transhistorique, trouve sa validité.)

Le point de départ de l'analyse critique ne peut donc trouver appui de manière cartésienne, dans une vérité supposément indubitable et valide transhistoriquement. Au contraire, le point de départ doit être historiquement spécifique, sur la base d'une analyse historiquement déterminée de la formation historiquement spécifique qu'est son contexte. Si Hegel, dans La science de la logique était préoccupé par le problème du point de départ pour l'exposition d'une logique qui ne présuppose pas une logique, c'est-à-dire un appui en-dehors de ce qu'il cherche à démontrer, Marx était lui préoccupé par la question du point de départ historiquement spécifique pour une théorie critique de la société qui ne s'appuierait pas sur autre chose que son objet/contexte.

Parce qu'un tel point de départ ne peut s'appuyer sur aucune proposition transhistoriquement valide, elle ne peut être rendue plausible que de manière immanente - par l'enchainement du dévoilement, où chaque moment successivement dévoilé justifie rétroactivement ce qui l'a précédé. C'est bien-sûr ainsi qu'est structuré Le Capital. Les catégories exposées au début - par exemple la marchandise, la valeur, la valeur d'usage, le travail concret, le travail abstrait - ne trouvent leur validité que dans le développement de l'analyse ${ }^{12}$. Ce qui apparaît être leur fondement transhistorique dans le premier chapitre du Capital doit être interprété à travers le mode de présentation rigoureusement immanent de Marx, qui n'a pas de point-de-vue extérieur à son objet. Compris ainsi, ce qui apparaît être une base transhistorique (de la valeur, par exemple) n'est que la manière dont les formes objectives/subjectives se présentent elles-mêmes. C'est un métacommentaire sur la pensée qui reste soumis aux limites des formes structurantes de la société moderne et capitaliste ${ }^{13}$.

\section{Le Capital à la lumière des Grundrisse}

Arrivé à ce point, nous pouvons brièvement esquisser une lecture du Capital à partir des idées développées jusqu'ici. Comme on le sait, le point de départ du Capital est la marchandise. Sur la base des Grundrisse, il est maintenant évident que la catégorie de marchandise ne se réfère pas ici aux marchandises telles qu'elles existent dans de nombreuses sociétés. Elle n'exprime pas non plus une étape historique (qui relèverait de la fiction) dans laquelle la « production de marchandises simples » seraient antérieure au capitalisme. Au contraire, la catégorie de marchandise est ici historiquement spécifique. Elle désigne la forme sociale la plus fondamentale dans la société capitaliste, la forme à partir de laquelle Marx s'applique à dévoiler les éléments essentiels et la particularité de 
la dynamique de cette société ${ }^{14}$. Les caractéristiques de cette forme - qui est à la fois une valeur et une valeur d'usage, par exemple - doivent également être comprises comme historiquement spécifiques (Marx 1996 : 84, 87).

En tant que forme de relations sociales, selon Marx, la marchandise est particulière : elle est constituée par le travail. Par conséquent, elle existe nécessairement dans une forme objectivée et possède un caractère dual en tant que forme de médiation sociale et en tant que produit, comme valeur et comme valeur d'usage. La conception, par Marx, de la spécificité historique du travail dans le capitalisme souligne cette description. Il maintient que le travail à un double caractère dans le capitalisme : il est à la fois «travail concret » et «travail abstrait " (Marx 1996: 51-56). Le "travail concret» se réfère aux activités de médiation de l'interaction entre les hommes et la nature. Bien que ce ne soit que dans le capitalisme que ces activités soient centrales et dominantes, et que tous les produits soient classés de la même manière, en tant que valeurs d'usage, cette sorte d'activité de médiation est transhistorique, elle existe dans toutes les sociétés. La valeur d'usage de la marchandise n'est pas historiquement propre au capitalisme. Cela implique toutefois que sa dimension de valeur et le travail qui la constitue sont historiquement spécifiques. Ainsi, le «travail abstrait » n'est pas le travail concret en général, mais est une catégorie différente et historiquement spécifique. Comme argumenté dans Temps, travail et domination sociale, cela signifie que le travail dans le capitalisme a une fonction sociale unique qui n'est pas intrinsèque aux activités de travail en tant que telles (Postone 1993 : 123-185). Au contraire, le travail déterminé par la marchandise devient un moyen quasi-objectif avec lequel les produits des autres sont acquis. Cela médiatise une nouvelle forme d'interdépendance, où le travail des gens, ou bien les produits du travail, fonctionnent comme des moyens quasi-objectifs pour obtenir les produits des autres. En devenant de tels moyens, le travail et ses produits préemptent cette fonction des relations sociales manifestes.

Alors, dans les travaux ultérieurs de Marx, la notion de la centralité essentielle du travail dans la vie sociale est historiquement spécifique. Cela ne veut pas dire que la production matérielle est la dimension la plus essentielle de la vie sociale en général, ou du capitalisme en particulier. Cela renvoie plutôt à la construction historiquement spécifique d'une forme de médiation par le travail qui caractérise fondamentalement cette société. Toutefois, cette activité de médiation n'est pas une caractéristique intrinsèque à l'activité de travail. Par conséquent, elle n'apparaît pas comme telle - et ne le peut pas. En revanche, quand la marchandise est analysée, sa dimension historiquement spécifique, la valeur, apparaît comme étant constituée par le travail en général, sans plus de qualification - «la dépense de cervelle, de nerf et de muscle humain» (Marx 1996: 54). Cela signifie que la fonction de médiation sociale historiquement spécifique du travail dans le capitalisme semble être du travail concret transhistorique, du «travail » - c'est-à-dire comme une essence ontologique plutôt que comme une forme historiquement spécifique. Cette forme apparemment ontologique du travail, alors qu'il est propre au capitalisme dans ses fonction de constitution sociale, est une détermination fondamentale de ce que Marx appelle les formes fétiches du capitalisme. Cela contredit les approches qui transhistorisent le rôle socialement constituant du travail dans le capitalisme, que ce soit positivement (comme l'économie politique classique et le marxisme traditionnel) ou négativement (comme dans la Dialectique de la raison $)^{15}$. 
40 Alors, le travail dans le capitalisme ne médiatise pas seulement les interactions des humains et de la nature, mais constitue également une médiation sociale historiquement spécifique, selon Marx. Ainsi, ses objectivations (la marchandise, le capital) sont à la fois des produits du travail concret et des formes objectivées de médiation sociale. Suivant cette analyse, les relations sociales qui caractérisent fondamentalement les formes capitalistes de la vie sociale sont différentes des relations spécifiquement qualitatives et manifestes, telles que les liens de parentés, qui caractérisent d'autres formes de vie sociale. Les formes de relations sociales fondamentales constitutives du capitalisme sont particulièrement quasi-objectives et formelles, et se caractérisent par l'opposition dialogique entre une dimension abstraite, générale et homogène, et une dimension concrète, particulière et matérielle (qui apparaissent toutes deux comme étant naturelles et non sociales).

41 Cette forme historiquement spécifique de médiation est constituée par des formes déterminées de pratiques, mais elle devient quasiment indépendante de ces dernières. Le résultat est une nouvelle forme de domination sociale qui assujetti les gens à des contraintes et des impératifs impersonnels «rationnels » qui ne peuvent être saisis adéquatement en termes de domination concrète de groupes sociaux, qu'ils soient une classe ou des agents institutionnels de l'État et/ou de l'économie. À l'instar du pouvoir conceptualisé par Foucault, cette forme de domination n'émane pas d'un lieu déterminé et n'apparaît absolument pas comme étant sociale. Cependant, elle n'est pas statique mais elle est temporellement dynamique. Dans Le Capital, Marx traite le caractère historiquement dynamique du capitalisme comme étant déterminé historiquement, spécifiant la caractéristique de cette forme de vie sociale, enraciné dans la forme de domination impersonnelle intrinsèque aux formes de base structurant cette société. Ce faisant, il relativise historiquement la notion d'une dynamique historiquement intrinsèque.

Ce qui régit cette dynamique est le double caractère des formes sociales sous-jacentes au capitalisme. À cet égard, il est éminemment important de noter que la distinction faite par Marx dans les Grundrisse entre la valeur et la « richesse réelle » réapparait dans le premier chapitre du Capital dans la distinction entre la valeur et la « richesse matérielle » (Marx 1996: 53-56). La richesse matérielle se mesure par la quantité produite, et est fonction de nombreux facteurs en plus du travail, tels que le savoir, l'organisation sociale et les conditions naturelle (Marx 1996: 50). La valeur, qui est la forme dominante de richesse dans le capitalisme, est uniquement constituée par la dépense de temps de travail humain (socialement nécessaire), selon Marx (Marx 1996 : 49-50, 55-56). Alors que la richesse matérielle, en tant que forme dominante de richesse, est médiatisée par des relations sociales manifestes, la valeur est une forme de richesse auto-médiatisante.

En commençant à traiter l'amplitude de la valeur en terme de temps de travail socialement nécessaire, Marx décrit l'interaction dynamique entre la valeur et la valeur d'usage qui devient historiquement signifiante avec l'émergence de la plus-value relative et donne naissance à une dynamique historique très complexe, et non linéaire, soutenant la société moderne. En décryptant cette dynamique, il devient de plus en plus clair que la forme historiquement spécifique de domination sociale, intrinsèque aux formes sociales les plus basiques du capitalisme, est la domination des gens par le temps. Cette dynamique esquissée par Marx dans Le Capital est caractérisée, d'une part, par des transformations constantes de la production et plus généralement de la vie sociale; et d'autre part, cette dynamique historique entraine la reconstitution constante de sa 
propre condition fondamentale en élément de la vie sociale invariable - étant donné que la médiation sociale est au final réalisée par le travail et donc, que le travail vivant reste partie intégrante du processus de production (considéré en tant que société dans son ensemble) quel que soit le niveau de productivité. Le capitalisme génère sans cesse du nouveau tout en reconstituant toujours la même chose.

Cette compréhension de la dynamique complexe du capitalisme ouvre la voie pour la critique, à une analyse sociale (plutôt que technologique) de la trajectoire de la croissance et de la structure de la production dans la société moderne. Bien que je ne puisse approfondir ici, le concept clé de plus-value chez Marx n'indique pas seulement, comme le souligne l'interprétation traditionnelle, que le surplus est produit par le classe laborieuse, mais que le capitalisme se caractérise par une forme déterminée d'emballement de la croissance. Le problème de la croissance économique dans le capitalisme, suivant cette grille de lecture, n'est pas seulement sa tendance à la crise, comme cela a été souligné fréquemment et correctement par les approches marxistes traditionnelles. C'est plutôt que la forme de la croissance elle-même, qui entraine la destruction accélérée de l'environnement naturel pour une augmentation toujours moindre de plus-value, est en elle-même problématique. La trajectoire de la croissance serait différente, selon cette approche, si le but ultime de la production était l'augmentation de la quantité de biens plutôt que l'augmentation de la plus-value.

Cette approche fournie également les bases pour une analyse critique de la structure du travail social et de la nature de la production dans le capitalisme. Elle indique que le processus de production industrielle ne devrait pas être compris comme un procédé technique qui, bien que toujours plus socialisé, est utilisé par les capitalistes privés à leurs propres fins. L'approche que je dessine comprend plutôt que le procédé lui-même est intrinsèquement capitaliste. Avec la subsomption réelle du travail, selon Marx, le capital devient de moins en moins la forme mystifiée du pouvoir qui serait « en fait » celui des travailleurs. Au contraire, les pouvoirs productifs du capital deviennent les pouvoirs productifs socialement généraux qui ne peuvent plus être compris adéquatement comme ceux des seuls producteurs immédiats. Cette constitution et cette accumulation du savoir socialement général rendent le travail prolétarien de plus en plus anachronique. C'est-àdire qu'elles rendent la production de la richesse matérielle essentiellement indépendante de la dépense de temps de travail humain direct. Partant, cela ouvre la possibilité de réductions à grande échelle du temps de travail et de changements fondamentaux de la nature et de l'organisation sociale du travail. Ceci dit, ces possibilités ne sont pas et ne peuvent pas se réaliser dans le capitalisme : la dialectique de la valeur et de la valeur d'usage reconstitue la nécessité du travail prolétarien. La combinaison de la course du capital pour une augmentation croissante de la productivité avec son enracinement dans la dépense de temps de travail humain direct, mène à un mode de production déterminé, dans lequel le développement de la production technologiquement sophistiquée qui pourrait libérer les gens d'une activité fragmentée et répétitive, renforce au contraire un tel travail. De la même manière, le temps de travail n'est pas réduit à un niveau socialement général mais est distribué de manière inégale, augmentant même pour certains.

Cette présentation préliminaire de la notion de contradiction capitaliste chez Marx indique que son analyse cherche à saisir la course du développement capitaliste comme un développement à double tranchant, d'enrichissement d'une part et d'appauvrissement de l'autre. Cela implique que ce développement ne peut être compris de manière 
adéquate sur un mode unidimensionnel, ni comme le progrès du savoir et du bonheur, ou comme le « progrès » de la domination et de la destruction. Selon son analyse, bien que la possibilité historique fasse apparaitre que la modalité du travail social pourrait être enrichissante pour chacun, le travail social est devenu vraiment l'appauvrissement pour le plus grand nombre. Alors, l'augmentation rapide des savoirs scientifiques et techniques dans le capitalisme ne signifie pas un progrès linéaire vers l'émancipation. Selon l'analyse de la marchandise et du capital par Marx, un tel savoir croissant - lui-même socialement constitué - a mené à la fragmentation et la perte de sens du travail individuel, et au contrôle croissant de l'humanité par le produit de sa propre activité objectivée. Il a alors également accentué la perspective d'une activité individuellement enrichissante et d'un plus grand contrôle de l'humanité sur son devenir. Ce développement à double tranchant est enraciné dans les structures aliénées de la société capitaliste et peut être dépassé selon l'analyse dialectique de Marx. Celle-ci ne peut alors, en aucun cas, être assimilée à une foi dans le progrès scientifique linéaire et/ou dans le progrès social.

L'analyse de Marx implique donc une idée du dépassement du capitalisme qui n'affirme ni de manière acritique la production industrielle comme étant la condition du progrès humain, ni le rejet romantique du progrès technologique en soi. En indiquant que le potentiel du système de production développé sous le capitalisme pourrait être utilisé pour transformer ce système, l'analyse de Marx dépasse l'opposition de ces positions, et indique que chacune d'entre elles considère le mouvement unique d'un développement historique complexe comme étant ce développement dans son ensemble. Cette approche comprend l'opposition entre la foi dans le progrès linaire et son rejet romantique comme l'expression d'un antagonisme historique qui, dans les deux cas, est caractéristique de l'ère capitaliste. Plus généralement, la théorie critique de Marx ne soutient ni la simple conservation ni l'abolition de ce qui constitue historiquement le capitalisme. Sa théorie met plutôt l'accent sur une possibilité : ce qui a été historiquement constitué dans une forme aliénée pourrait être appropriée, et donc, fondamentalement transformée.

Suivant la très brève interprétation proposée ici, les Grundrisse nous permettent de voir que la critique élaborée par Marx dans Le Capital va bien plus loin que la critique traditionnelle des relations de distributions bourgeoises (le marché et la propriété privée). Elle n'implique pas seulement une critique de l'exploitation et de la distribution inégale de la richesse et du pouvoir, même si bien-sûr, elle inclut une telle critique. Elle comprend plutôt la société industrielle moderne comme étant en elle-même capitaliste, et analyse de manière critique le capitalisme d'abord en terme d'abstraction des structures de domination, de fragmentation croissante de l'activité et de l'existence individuelles, et d'une logique de développement à la course aveugle. Elle traite la classe laborieuse comme étant l'élément de base du capital, plutôt que comme la réalisation de sa négation, et conceptualise implicitement le socialisme non pas en termes de libération du travail et de la production industrielle, mais en termes de la possible abolition du prolétariat et de l'organisation du travail basée sur le travail prolétarien (tout comme du système dynamique de compulsion abstraite constitué par le travail en tant qu'activité de médiation sociale). Cette approche ré-conceptualise une société postcapitaliste dans les termes du dépassement du prolétariat - l'auto-abolition du prolétariat et de son travail c'est-à-dire en termes de transformation des structures générales du travail et du temps. En ce sens, elle diffère à la fois de la notion marxiste traditionnelle de "réalisation » du prolétariat, et du mode capitaliste d'abolition de la classe ouvrière nationale en créant 
une sous-classe dans le cadre d'une distribution inégale du travail et du temps, nationalement et mondialement.

Bien que le niveau logiquement abstrait de l'analyse décrite ici n'aborde pas directement la question des facteurs spécifiques soutenant les transformations structurelles des 30 dernières années, elle fournie un cadre à travers lequel ces transformations peuvent être abordées socialement et comprises historiquement. Elle pourrait aussi fournir la base pour une théorie critique du "socialisme réellement existant » comme forme alternative à l'accumulation capitaliste, plutôt que comme une modalité sociale qui représenterait la négation historique du capital, bien que de manière imparfaite. Dans la mesure où elle cherche des racines sociales, et où elle est une critique des relations sociales abstraites quasi-objectives, de la nature de la production, du travail et des impératifs de croissance dans le capitalisme, cette approche pourrait commencer à éclairer de nombreuses préoccupations contemporaines, d'aspirations et de mécontentements liés au développement du capital, même si elle ne le ferait pas nécessairement en termes traditionnels de classes sociales.

Ainsi, cette lecture de Marx cherche à contribuer à une compréhension critique des transformations globales de notre univers social, qui dépasserait les faiblesses des discours post-marxistes tout en évitant les pièges des approches du marxisme traditionnel.

\section{BIBLIOGRAPHIE}

Adorno, Theodor and Horkheimer, Max (2002) Dialectic of Enlightenment, Stanford: Stanford University Press.

Althusser, Louis and Balibar, Etienne (1970) Reading Capital, London: NLB.

Bell, Daniel (1973) The Coming of Post-Industrial Society, New York: Basic Books.

Cohen, G.A. (1986) 'Forces and Relations of Production', in J. Roemer (ed.), Analytical Marxism, Cambridge: Cambridge University Press.

Cohen, G.A. (1988) History, Labour and Freedom, Oxford: Clarendon Press.

Dobb, Maurice (1940) Political Economy and Capitalism, London: G. Routledge \& Sons.

Elster, Jon (1985) Making Sense of Marx, Cambridge: Cambridge University Press.

Giddens, Anthony (1979) Central Problems in Social Theory, Berkeley: University of California Press.

Habermas, Jürgen (1973) 'Between Philosophy and Science: Marxism as Critique', in Jürgen

Habermas, Theory and Practice, Boston: Beacon Press.

Marx, Karl (1973) Grundrisse: Foundations of the Critique of Political Economy, Harmondsworth: Penguin.

Marx, Karl (1987) 'A Contribution to the Critique of Political Economy', in Marx and Engels Collected Works, vol. 29: Marx 1857-61, New York: International Publishers. 
Marx, Karl (1994) 'Results of the Direct Process of Production', in Marx and Engels Collected Works, vol. 34: Marx 1861-64, New York: International Publishers.

Marx, Karl (1996) 'Capital, vol. I’, in Marx and Engels Collected Works, vol. 35: Capital, Vol. I, New York: International Publishers.

Meeks, Ronald (1956) Studies in the Labour Theory of Value, New York and London: Lawrence and Wishart.

Murray, John Patrick (1979) ‘Enlightenment Roots of Habermas' Critique of Marx', The Modern Schoolman, 57(1).

Negri, Antonio (1989) Marx Beyond Marx: Lessons on the Grundrisse, New York: J.F. Bergin Publishers.

Postone, Moishe (1993) Time, Labour, and Social Domination: A Reinterpretation of Marx's Critical Theory, Cambridge and New York: Cambridge University Press.

Roemer, John (1981) Analytical Foundations of Marxian Economic Theory, Cambridge: Cambridge University Press.

Rosdolsky, Roman (1977) The Making of Marx's Capital, London: Pluto Press.

Steedman, Ian (1981) 'Ricardo, Marx, Sraffa', in Ian Steedman (ed.), The Value Controversy, London: NLB.

Sweezy, Paul (1968) The Theory of Capitalist Development, New York: Oxford University Press.

\section{NOTES}

1. Pour éviter les malentendus par le terme « catégorique ", j'utilise celui de " catégoriel » pour renvoyer à l'ambition de Marx de saisir les formes de la vie sociale moderne à travers les catégories élaborées dans sa critique ultérieure.

2. Certains des arguments présentés ici sont développés dans Temps, travail et domination sociale.

3. Voir par exemple, G.A. Cohen, History, Labour and Freedom (Cohen 1998 : 209-238); Maurice Dobb, Political Economy and Capitalism (Dobb 1940 : 70-78) ; Ronald Meeks, Studies in the Labour Theory of Value (Meeks 1956); John Roemer, Analytical Foundations of Marxian Economic Theory (Roemer 1981 : 158-159) ; Ian Steedman, «Ricardo, Marx, Sraffa » (Steedman 1981 : 11-19) ; Paul Sweezy, The Theory of Capitalist Development (Sweezy 1968 : 52-53).

4. Y compris les interprétations les plus récentes de Marx - le structuralisme et la Théorie Critique. Althusser, par exemple, a formulé une critique tranchante et épistémologiquement sophistiquée du «travail idéalisé » et de la conception des gens en tant que sujet; il introduisait l'idée que les relations sociales sont des structures irréductibles à l'inter-subjectivité anthropologique. Pourtant, sa focalisation sur la question du surplus en terme d'exploitation, tout comme sur la dimension physique «matérielle» de la production, renvoient indubitablement à une compréhension traditionnelle du capitalisme (Althusser et Balibar 1970 : 145-154, 165-182). Lukács et les membres de l'École de Francfort, cherchant à interpréter théoriquement les transformations du capitalisme d'une forme centrée sur le marché à une forme bureaucratique centrée sur l'État, reconnaissaient tacitement les inadéquations d'une théorie critique de la modernité ne définissant le capitalisme que dans les termes du dixneuvième siècle - c'est-à-dire en termes de marché et de propriété privée des moyens de production. D'un autre côté pourtant, ils restaient soumis à certaines hypothèses de cette théorie (voir Postone 1993 : 71-120). 
5. John Stuart Mill, Principals of Political Economy (seconde édition, London 1849), volume 1, pp. 239-240 (cité dans Marx, 1973 : 832).

6. Jon Elster fourni un exemple d'un tel argument. Il contredit la théorie de la valeur et de la plus-value de Marx en déniant que « que les travailleurs ont une capacité mystérieuse à créer exnihilo »; il soutient au contraire que «la capacité de l'homme à se servir de l'environnement rend possible un surplus dépassant tout niveau de consommation donné » (Elster 1985 : 141). En posant la question de la création de richesse de cette manière, l'argument d'Elster traite implicitement la valeur en catégorie transhistorique, éludant ainsi la distinction faite par Marx entre « valeur » et « richesse réelle».

7. L'idée selon laquelle le valeur pour Marx n'est pas une catégorie de la richesse en général, mais qu'elle spécifie la forme de richesse et les relations sociales au cœur du capitalisme, a été incomprise par des penseurs aussi différents que Jürgen Habermas, Daniel Bell et Antonio Negri. Bell et Habermas affirmaient au débat des années 70, que la théorie de la valeur par rapport au travail avait été annulée historiquement et que la société contemporaine nécessitait une "théorie de la valeur par rapport à la science et la technologie». Tous deux réfutaient alors la distinction faite par Marx entre valeur et "richesse réelle ", et donc également la dynamique dialectique qu'il développait (Habermas 1973: 222-229); (Bell 1973: XIV). Negri quant à lui arguait que la description faite par Marx de ce que j'ai montré comme étant une organisation postcapitaliste, décrivait en fait le capitalisme contemporain. Celui-ci ne serait plus basé sur la loi de la valeur mais sur la «loi de l'ordre » (Negri 1989: 144ff.). De telles positions substituent implicitement une perception linéaire de l'histoire à l'analyse dialectique du nécessaire et du superflu faite par Marx.

8. L'idée que la contradiction de base du capitalisme est, selon Marx, structurel et ne renvoie pas simplement à l'antagonisme social a également été développée par Anthony Giddens. Cependant, ce dernier situe la contradiction dans l'antagonisme entre l'appropriation privée et socialisée de la production, c'est-à-dire, entre les relations bourgeoises de distribution et la production industrielle (Giddens 1979 : 135-141).

9. Pour un tel développement, voir G.A. Cohen, « Forces and Relations of Production » (Cohen $1986: 19-22)$.

10. En dépit de leurs différences, Georg Lukács, Theodor Adorno et Alfred Sohn-Rethel reconnaissent le caractère objectif/subjectif des catégories de Marx, rompant ainsi avec le schéma infra/superstructure.

11. Un des nombreux accomplissement du Capital fut d'enraciner socialement la projection transhistorique des catégories valides seulement dans la société capitaliste dans toutes les formes de la vie sociale humaine. Marx le fit en basant ces projections dans les nombreuses formes fétichisées des catégories, qui sont générées par le jeu entre l'abstraction particulière et les dimensions concrètes des formes de médiations sociales constitutives de la société capitaliste.

12. Cela est développé dans Temps, travail et domination sociale.

13. Voir John Patrick Murray, "Enlightenment Roots of Habermas' Critique of Marx», The Modern Schoolman, 57, $n^{\circ} 1$ (November 1979), pp. $13 \mathrm{ff}$.

14. Roman Rosdolsky a remarqué que l'existence du capital développé est énoncé au tout début de la critique élaborée par Marx (Rosdolsky 1977 : 46).

15. Voir Theodor Adorno et Max Horkheimer, Dialectique de la Raison (Adorno et Horkheimer 2002). 
INDEX

Mots-clés : critique du travail, correspondance, artistes, socialisme vert, travailleurs culturels, Wertkritik, Gorz André, dialogue, Vincent Jean-Marie, écologie, modèles alternatifs, Marx Karl, penser le Capital, ethos du travail, Eglise Orthodoxe, salariat, domination

\section{AUTEURS}

\section{MOISHE POSTONE}

Professeur d'histoire à l'Université de Chicago, membre du Committee on Jewish Studies. Auteur notamment de Temps, travail et domination sociale (2009, Mille et Unes Nuits, 587 p.). 